\title{
ON FOURIER COEFFICIENTS OF EISENSTEIN SERIES ${ }^{1}$
}

\author{
BY LIANG-CHI TSAO \\ Communicated by Calvin C. Moore, December 4, 1972
}

In this paper we wish to prove that under certain conditions the Fourier coefficients of the Eisenstein series for an arithmetic group acting on a tube domain are all rational numbers.

Let $G$ be a connected, simply-connected, semisimple, and almost $\boldsymbol{Q}$-simple linear algebraic group defined over the rational number field $\boldsymbol{Q}$. Let $\boldsymbol{R}$ be the real number field. Then $G_{\boldsymbol{R}}$ is connected, and we assume that if $K$ is a maximal compact subgroup of it, then $\mathfrak{X}=K \backslash G_{R}$ is a noncompact hermitian symmetric space. We also assume that the $\boldsymbol{Q}$-rank of $G$ is positive and that the $Q$-root system ${ }_{Q} \Sigma$ of $G$ is of type C. Then the relative $\boldsymbol{R}$-root system ${ }_{\boldsymbol{R}} \Sigma$ of $G$ is of type $\mathrm{C}$ and therefore $\mathfrak{X}$ is isomorphic to a tube domain

$$
\mathfrak{I}=\left\{X+i Y \in \boldsymbol{C}^{m} \mid Y \in \mathfrak{R}\right\},
$$

where $\boldsymbol{C}$ is the complex number field, and $\boldsymbol{R}$ is a homogeneous, selfadjoint cone in $\boldsymbol{R}^{\boldsymbol{m}}$. Moreover, it follows that an isomorphism of $\mathfrak{X}$ with $\mathfrak{I}$ may be chosen such that

(2) $P_{\boldsymbol{R}}=\left\{g \in G_{\boldsymbol{R}} \mid g\right.$ acts by linear affine transformation on $\left.\mathfrak{I}\right\}$,

then $P_{R}$ is a $Q$-parabolic subgroup of $G_{R}$, and every element of the unipotent radical $U_{\boldsymbol{R}}$ of $P_{\boldsymbol{R}}$ acts by a real translation. Finally, we may write $G=R_{K / Q} G^{*}$, where $G^{*}$ is absolutely almost simple, and defined over a totally real algebraic number field $K$, and $R_{K / Q}$ is the ground field reduction functor [9, Chapter 1$]$.

Let $\Gamma \subset G_{R}$ be an arithmetic subgroup of $G_{C}$, then $\Lambda^{\prime}=\Gamma \cap U$ is a lattice in $U_{\boldsymbol{R}}$. If $F$ is an automorphic form of even weight $l$ with respect to $\Gamma$, i.e.,

$$
F(Z \cdot \gamma) j(Z, \gamma)^{l}=F(Z), \quad \text { for all } \gamma \in \Gamma,
$$

AMS (MOS) subject classifications (1970). Primary 10D20; Secondary 10G05, 32N99.

Key words and phrases. Eisenstein series, tube domain, linear algebraic group, hermitian symmetric space, homogeneous selfadjoint cone, parabolic subgroup, arithmetic subgroup, automorphic form, functional determinant, lattice, Jordon algebra, determinant, rank, boundary component, Gamma integral, Poisson summation formula, content, exponential sum, Gaussian sum, $L$-function.

${ }^{1}$ The author wishes to thank his thesis advisor, Professor W. L. Baily, Jr., for his assistance throughout this work. 
where $j(Z, g)$ is the functional determinant of $g \in G_{\boldsymbol{R}}$ at $Z \in \mathfrak{I}$, then

$$
F(Z+S)=F(Z), \text { for all } S \in \Lambda^{\prime} .
$$

Therefore we have the Fourier expansion:

$$
F(Z)=\sum_{T \in \Lambda} a(T) e^{2 \pi i(T, Z)},
$$

where $\Lambda$ is the dual lattice of $\Lambda^{\prime}$ with respect to a nondegenerate symmetric bilinear form (, ) on $U$, with respect to which $\Omega$ is selfadjoint.

If $a \in G_{Q}$, define ${ }^{a} P=a P a^{-1}$, and $\Gamma_{0, a}=\Gamma \cap{ }^{a} P, \Gamma_{0}=\Gamma_{0, e}=\Gamma \cap P$. Since $P$ is a $Q$-parabolic subgroup, we have $G_{Q}=\bigcup_{a \in A} \Gamma a P_{Q}, A \subset G_{Q}$ being a finite set.

For each $a \in G_{Q}$, we may form an Eisenstein series

$$
E_{l, a}(Z)=\sum_{\gamma \in \Gamma / \Gamma_{0, a}} j(Z, \gamma a)^{l}
$$

it is an automorphic form of weight $l$ with respect to $\Gamma$.

Now, for each $a \in G_{Q}$, we may choose a number $c(a)$ and form the Eisenstein series

$$
E_{l}(Z)=\sum_{a \in A} E_{l, a}(Z) c(a)^{l}
$$

We explain briefly how the $c(a)$ are chosen. Namely, we first consider Eisenstein series on the adele group $G_{A}$ of $G$ and then view these as automorphic forms on $\mathfrak{I}$. The result is linear combinations of the form (7), and these linear combinations are independent of the choice of the set $A$ of representatives, whence a definition of $c(a)$ for every $a \in G_{Q}$.

The main object of this work is to see that if $c(a)$ are chosen in this way, then the Fourier coefficients $a_{l}(T)$ of the Fourier expansion of $E_{l}(Z)$,

$$
E_{l}(Z)=\sum_{T \in \Lambda} a_{l}(T) e^{2 \pi i(T, Z)}
$$

are all rational numbers.

Tube domains. Any tube domain is a direct product of irreducible tube domains. The irreducible tube domains may be divided into the following five types:

A. $R$ is the cone of $n \times n$ positive definite hermitian symmetric matrices $\left(m=m^{2}\right)$.

B. $\Omega$ is the cone of $n \times n$ positive definite real symmetric matrices $(m=n(n+1) / 2)$.

C. $\Omega$ is the cone of $n \times n$ positive definite quaternion hermitian symmetric matrices $(m=n(2 n-1))$. 
D. $\boldsymbol{R}$ is the cone of the points $\left(x_{1}, \ldots, x_{n}\right)$ in the $n$-dimensional real vector space such that $x_{1}^{2}>\sum_{i=2}^{n} x_{i}^{2}, x_{1}>0(m=n)$.

E. $\boldsymbol{R}$ is the cone of $3 \times 3$ positive definite Cayley hermitian symmetric matrices $(m=27)$.

Example and historical background. Let $\mathfrak{I}$ be Siegel's generalized half plane of degree $n$; it is the irreducible tube domain of type B for this $n$. We have $G_{\boldsymbol{R}}=\operatorname{Sp}(n, \boldsymbol{R})$. If $\left(\begin{array}{cc}A & B \\ D\end{array}\right) \in \operatorname{Sp}(n, \boldsymbol{R})$, and $Z \in \mathfrak{I}$, then

$$
Z \cdot\left(\begin{array}{ll}
A & B \\
C & D
\end{array}\right)=(Z B+D)^{-1}(Z A+C) \text {. }
$$

We have

$$
\begin{aligned}
P_{\boldsymbol{R}} & =\left\{\left(\begin{array}{cc}
A & 0 \\
{ }^{t} A^{-1} S & { }^{t} A^{-1}
\end{array}\right) \mid S \text { :symmetric }\right\}, \\
U_{\boldsymbol{R}} & =\left\{\left(\begin{array}{ll}
1 & 0 \\
S & 1
\end{array}\right) \mid S \text { : symmetric }\right\} .
\end{aligned}
$$

If we let $\Gamma=\operatorname{Sp}(n, Z)$, the symplectic modular group, then $\Lambda^{\prime}=$ integral symmetric matrices, $\Lambda=$ half-integral symmetric matrices. The finite set $A$ contained in $G_{Q}$ in this case is $\{e\}$.

C. Siegel [8] proved the rationality of $a_{l}(T)$ in the above case in 1939. H. Braun [4] dealt with some special cases of the tube domains of type $A$ in 1949, and H. Klingen [6] dealt with the Hilbert modular group (a special case of type B) in 1960, and then W. Baily, Jr. dealt with an irreducible case of type $\mathrm{E}$ in 1970 [3].

Many ideas of the proof, in this paper, of the same result for a more general class of tube domains and arithmetic subgroups come from the ideas of the proof in that paper by W. Baily, Jr.

We now sketch the order of developments in this paper. At first, we describe the relationships between tube domains and Jordan algebras [7], and then discuss the structures of Jordan algebras. In particular, we describe the $\boldsymbol{R}$ - and $\boldsymbol{K}$-Jordan algebra structures of $U$ as well as the notions of determinant and rank of a class of Jordan algebras. Later we need certain facts about parabolic subgroups, functional determinant and boundary components and the relationships among them, which enable us, by way of the Bruhat decomposition, to reduce our treatment of the Fourier coefficients, by induction, to those associated to the "biggest cell". That is, if $i$ is the element of $G_{\boldsymbol{R}}$ sending each $Z \in \mathfrak{T}$ to $-Z^{-1}$, then it suffices to treat the Fourier coefficients of

$$
E_{l}^{(n)}=\sum_{g \in P_{Q^{i P} Q^{/ P} Q}} j(Z, g)^{l} c(g)^{l}=\sum_{T \in \Lambda} a_{l}^{(n)}(T) e^{2 \pi i(T, Z)} .
$$


It is proved that $j(Z, i)=|Z|^{-N}$, where $|Z|$ is the determinant of $Z$ and $N=(n-1) n_{0}+2, n_{0}$ being equal to $2,1,4, n-2$ or 8 corresponding to types A, B, C, D or E respectively. Hence $E_{l}^{(n)}(Z)$ can be written as

$$
\left.E_{l}^{(n)}(Z)=\sum_{X \in U_{Q}} \mid Z+X\right)^{-N l} c\left(t_{X} i\right)^{l},
$$

where $t_{X}$ is the real translation by $X$.

Then, by applying the Gamma integral [5] and the Poisson summation formula (cf. $[8, \S 7],[3, \S 9.3]$ ), we have

$$
\begin{aligned}
a_{l}^{(n)}(T)= & v(\Lambda)^{-1}(2 \pi i)^{d n N l} \pi^{-d n(N-2) / 4} \prod_{j=0}^{n-1} \gamma\left(N l-j n_{0} / 2\right)^{-d} \\
& \cdot|T|^{N l-N / 2} \sum_{X \in U_{Q^{\prime}} \Lambda^{\prime}} e^{2 \pi i(T, X)} c\left(t_{X} i\right)^{l},
\end{aligned}
$$

where $d=[\boldsymbol{K}: \boldsymbol{Q}], \gamma(\quad)$ is the $\Gamma$-function and $v(\Lambda)$ is the volume of the fundamental set of $\Lambda$.

It remains to calculate $S=\sum_{X \in U / / \Lambda^{\prime}} e^{2 \pi i(T, X)} c\left(t_{X} i\right)^{l}$. We note that $c\left(t_{X} i\right)$ is the content of $X$ in Siegel's case. Following the same idea as in $([8, \S 7]$, $[3, \S 9.3]$ ), we write $S=\prod_{p} S_{p}, S_{p}=\prod S_{p}$, where $p$ runs over all finite prime numbers and $\mathfrak{p}$ over all prime ideals of $\boldsymbol{K}$ dividing $p$. Applying Hensel's lemma [1], we may prove that, for all prime ideals $\mathfrak{p}$ of $K, S_{\mathfrak{p}}$ is rational, and for all but a finite number of prime ideals $\mathfrak{p}$, we have

$$
S \mathfrak{p}=\sum_{X \in i_{p^{\alpha}}} \exp \left((2 \pi i / p) \operatorname{tr}_{F_{p^{\alpha}} / F_{p}}(T, X)\right)_{p^{-\alpha R}(x) N l}
$$

provided that the $\boldsymbol{K}$-structure of the Jordan algebra is of a certain form, which includes tube domains of types B, C, D and E and some cases of type A, where $\alpha=[\boldsymbol{K p}: \boldsymbol{Q} p]$, and $\mathfrak{i}_{p^{\alpha}}$ is the Jordan algebra over the finite field $\boldsymbol{E}_{p^{\alpha}}$ of $p^{\alpha}$ elements, obtained in a certain way from the $\boldsymbol{K}$-structure of the original Jordan algebra, and $R(X)$ is the rank of $X$.

We then proceed to the explicit calculation of exponential sums $S \mathfrak{p}$, with the help of the technical lemmas proved earlier. The final results are (for all but a finite number of prime ideals $p$ ):

(i) $n_{0}$ even. $S \mathfrak{p}=\prod_{k=0}^{n-1}\left(1-\left(\sigma G^{\alpha n_{0}}\right)^{k} p^{-\alpha N l}\right)$,

(ii) type $D, n_{0}$ odd. $S \mathfrak{p}=\left(1+\sigma G^{\alpha\left(n_{0}+1\right)}(|T| / p)_{\alpha} p^{-\alpha N l}\right)\left(1-p^{-\alpha N l}\right)$,

(iii) type $\mathrm{B}, n=2 r+1$.

$$
S \mathfrak{p}=\left(1-p^{-\alpha N l}\right) \prod_{k=1}^{r}\left(1-G^{4 k \alpha} p^{-2 \alpha N l}\right),
$$

(iv) type $\mathrm{B}, n=2 r$.

$$
S \mathfrak{p}=\left(1-p^{-\alpha N l}\right)\left(1+G^{2 \alpha n}(|T| / p)_{\alpha} p^{-\alpha N l}\right) \prod_{k=1}^{r-1}\left(1-G^{4 \alpha k} p^{-2 \alpha N l}\right),
$$


where $\boldsymbol{G}=\sum_{x \in \boldsymbol{F}_{p^{\alpha}}} \exp (2 \pi i / p) x^{2}$ is the Gaussian sum, $(a / p)_{\alpha}=1$ or -1 according as the number $a \in \boldsymbol{E}_{p^{\alpha}}$ is a square or a nonsquare, and $\sigma=(|f| / p)_{\alpha},|f|$ being the determinant of the quadratic form $f$ of the entries of the Jordan algebra $\mathfrak{i}_{p^{\alpha}}$.

By calculating $v(\Lambda)$ and using the values of $S p$ and of $L$-functions we are able to prove that the Fourier coefficients in the cases we are considering are all rational numbers.

\section{REFERENCES}

1. W. L. Baily, Jr., On Hensel's lemma and exponential sums, Global Analysis (a volume of assorted articles dedicated to K. Kodaira), University of Tokyo Press, 1969, 85-100. MR 40 \# 4271.

2. - Eisenstein series on tube domains, in problems of analysis: A symposium in honor of S. Bochner, Princèton University Press, 1970.

3. - An exceptional arithmetic group and its Eisenstein series, Ann. of Math. (2) 91 (1970), 512-549. MR 42 \#4674.

4. H. Braun, Hermition modular functions, Ann. of Math. (2) 50 (1949), 827-855. MR 11, 333.

5. B. A. Fuchs, Special chapters of the theory of analytic functions of several complex variables, Transl. Mat. Monographs, Vol. 14, Amer. Math. Soc., 1965.

6. K. Klingen, Eisensteinreihen zur Hilbertschen Modulgruppe n-ten Grades, Nachr. Akad. Wiss. Göttingen Math.-Phys. K1. II (1960), 87-104. MR 23 \# A356.

7. A. Korányi and J. Wolf, Realization of hermitian symmetric spaces as generalized halfplanes, Ann. of Math. (2) 81 (1965), 265-288. MR 30 \#4980.

8. C. L. Siegel, Einführung in die Theorie der Modulfunktionen n-ten Grades, Math. Ann. 116 (1939), 617-657. MR 1, 203.

9. A. Weil, Adèles and Algebraic Groups, Notes by M. Demazure and T. Ono, Institute for Advanced Study, Princeton, 1961.

Department of Mathematics, University of Chicago, Chicago, Illinois 60637

Current address: Institute for Advanced Study, Princeton, New Jersey 08540 